\title{
BMJ Open Elucidating the information exchange during interfacility care transitions: Insights from a Qualitative Study
}

\author{
Lianne Jeffs, ${ }^{1}$ Marianne Saragosa, ${ }^{1}$ Madelyn Law, ${ }^{2}$ Kerry Kuluski, ${ }^{3}$ Sherry Espin, ${ }^{4}$ \\ Jane Merkley, ${ }^{5}$ Chaim M Bell ${ }^{6}$
}

To cite: Jeffs L, Saragosa M, Law $\mathrm{M}$, et al. Elucidating the information exchange during interfacility care transitions: Insights from a Qualitative Study. BMJ Open 2017;7:e015400. doi:10.1136/ bmjopen-2016-015400

- Prepublication history and additional material is available. To view please visit the journal (http://dx.doi.org/ 10.1136/ bmjopen-2016-015400).

Received 9 December 2016 Revised 11 May 2017 Accepted 23 May 2017

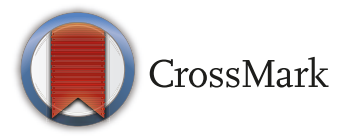

${ }^{1}$ St Keenan Research Centre of the Li Ka Shing Knowledge Institute, St Michael's, Toronto, Canada

${ }^{2}$ Department of Health Science, Brock University, St Michael's, Toronto, Canada

${ }^{3}$ Community Health Sciences, Lunenfeld-Tanenbaum Research Institute, Sinai Health System,

Toronto, Canada

${ }^{4}$ Daphne Cockwell School of Nursing, Ryerson University,

Toronto, Canada

${ }^{5}$ Executive Offices, Sinai Health System, Toronto, Canada ${ }^{6}$ Department of Medicine, University of Toronto, Toronto, Canada

Correspondence to

Dr Lianne Jeffs; jeffs|@smh.ca

\section{ABSTRACT}

Objective To explore the perceptions of patients, their caregivers and healthcare professionals associated with the exchange of information during transitioning from two acute care hospitals to one rehabilitation hospital.

Design An exploratory qualitative study using semistructured interviews and observation.

Participants and setting Patients over the age of 65 years admitted to an orthopaedic unit for a non-elective admission, their caregivers and healthcare professionals involved in their care. Participating sites included orthopaedic inpatient units from two acute care teaching hospitals and one orthopaedic unit at a rehabilitation hospital in an urban setting.

Findings Three distinct themes emerged from participants' narrative of their transitional care experience: (1) having no clue what the care plan is, (2) being told and notified about the plan and (3) experiencing challenges absorbing information. Participating patients and their caregivers reported not being engaged in an active discussion with healthcare professionals about their care transition plan. Several healthcare professionals described withholding information within the plan until they themselves were clear about the transition outcomes. Conclusion This study highlights the need to increase efforts to ensure that effective information exchanges occur during transition from acute care hospital to rehabilitation settings.

\section{INTRODUCTION}

It is well established that care transition points present potential for threats to safety and quality. ${ }^{1}$ These threats can result in harm to patients, ${ }^{2}$ an increased risk of readmission, ${ }^{3}$ increased length of stay ${ }^{4}$ and economic burden to the healthcare system and patients and their families. ${ }^{5}$ Deficits in communication and the inadequate exchange of information around discharge home from hospital ${ }^{16}$ or transfer to another healthcare facility ${ }^{28}$ underpin such threats. Poor information exchange often includes inaccurate or missing information regarding patients current health status, medication plan, functional and psychological history and unresolved plans for follow-up care

\section{Strengths and limitations of this study}

This study explores the understudied area of the perceptions of patients, their caregivers and healthcare professionals associated with the exchange of information during transitioning from two acute care hospitals to one rehabilitation hospital.

- The study was only conducted with non-elective orthopaedic patients and their caregiver at two acute care teaching hospitals and one rehabilitation hospital in an urban setting in Canada. Thus, our findings may not be transferrable to other geographical locations or other patient populations (eg, medically complex patients without orthopaedic injury).

- There is a potential for social desirability bias when conducting interviews with participants.

post discharge or transfer from a hospital to another facility. ${ }^{4}$ Patients and their caregivers (eg, family) may then be uninformed or misinformed about their illness and medications and not be able to carry out the care transitions plan or manage their own health. ${ }^{9}$

The exchange and transfer of accurate and complete information to patients and their caregivers around what is currently happening and what to expect next is an important component of ensuring quality care transitions. ${ }^{81011}$ By providing information to patients (and when available their caregivers), they are more informed and have the potential to actively participate in their care ${ }^{8}$ which is associated with improved patient satisfaction and clinical outcomes. ${ }^{12}$ However, varying degrees of information exchange with patients ${ }^{13}$ and their subsequent participation in their care planning ${ }^{11}{ }^{14}$ have been reported. The information exchange between acute care hospitals to rehabilitation settings has received less attention in the literature as compared with those discharged home from the hospital. ${ }^{7815}$ Minimal evidence in the literature exists around the nature 
of the information exchange ${ }^{15}$ including the patients' (and when available caregivers') desire for information. ${ }^{8}$ Insight is needed around the nature of information exchange with patients as they transition from acute care hospitals to rehabilitation settings, as these patients are potentially more vulnerable and their medical conditions more active than for patients discharged home.${ }^{15}$ Furthermore, this exchange of information often forms the basis for admission orders in the rehabilitation setting influencing subsequent transitions in care. ${ }^{15}$ In this context, a study was undertaken to explore the perceptions of patients, their caregivers and healthcare professionals associated with the exchange of information during transitioning from two acute care hospitals to one rehabilitation hospital.

\section{METHODS}

This study employed an exploratory qualitative design to gain insight into the perceptions and experiences associated with care transitions of non-elective patients 65 years and older, caregivers and healthcare professionals being transferred out of the acute care hospital to a rehabilitation facility. Non-elective patients did not expect to be admitted to hospital and therefore had no prehospital planning for transfer to another setting. Funding was received by the Ontario Ministry of Health and Long Term Care (grant number: 06693).

\section{Setting}

Participating sites included orthopaedic inpatient units from two acute care teaching hospitals and one orthopaedic unit at a rehabilitation hospital in an urban setting. Our study targeted patients 65 years and older who had undergone a non-elective admission (eg, a patient who had fallen or had an accident who had sustained a fracture), their family member (referred to here within as caregiver) and healthcare professionals (physicians, nurses, nurse practitioners, physiotherapists, occupational therapists, pharmacists and social workers).

\section{Recruitment and data collection process}

A purposeful sampling strategy was employed whereby study participants who met the eligibility criteria (see box 1) were identified and approached face to face by a

\section{Box 1}

Patient: 65 years and older, able to comprehend English and provide consent, and non-elective orthopaedic patient being transferred to specific rehabilitation facility.

Caregiver: caregiver of non-elective patient being transferred to specific rehabilitation facility who is 65 years and older and able to comprehend English and provide consent.

Healthcare professional: healthcare professionals at the acute care hospital or rehabilitation facility and able to able to comprehend English and provide consent and provided care to the patients who were recruited for the study. research coordinator (MS). ${ }^{16}$ The research coordinator was a master's prepared nurse with extensive experience in qualitative research. Eligible patients, who were unknown to the research coordinator prior to study commencement, were provided an overview of the study, and the research coordinator then obtained consent from those patients willing to participate. Once the patient was enrolled, the research coordinator approached caregivers at the participating sites for involvement in the study. Caregivers were provided an overview of the study, and then the research coordinator obtained their consent. Patient and caregiver interviews took place at the rehabilitation facility following the transfer from the acute care hospital. Most interviews were conducted face to face; however, for three caregivers, a total of four interviews (one caregiver was interviewed twice) took place over the phone for convenience.

Healthcare professionals were informed of the study by their respective unit managers. Members of the interprofessional team were selected based on their direct involvement in the care transition of the study patient at either the acute care or rehabilitation site. Those involved were approached face to face by the research coordinator who then provided an overview of the study and obtained informed consent prior to conducting the interview. Healthcare professionals consisted of nurses, social workers, occupational therapists, physical therapists, pharmacists, physicians and unit managers. Healthcare professionals were interviewed face to face either at the acute care site or the rehabilitation site following the transition of the patient.

Semi-structured interview guides were used with study participants to elicit their perceptions and experiences associated with the care transitions of non-elective medically complex patients 65 years and older. The interview guides were developed from a realist review and Delphi panel detailed in a separate paper ${ }^{17}$ and are included as online supplementary file 1 and supplementary file 2. Pilot testing of the interview guide occurred with the first few participants with additional prompts made to the interview guides. Patients and caregivers were interviewed after they transitioned from the rehabilitation hospital from the acute care and where possible prior to being discharged home from the rehabilitation organisation. This occurred in six incidences where patients or their caregiver were interviewed either shortly before or after discharge home. Healthcare professions participated in one interview. All interviews were audio recorded and transcribed verbatim by a professional transcriptionist. Field notes were taken by the research coordinator following the interview and shared with the research team. Duration of interviews averaged between 6 and $60 \mathrm{~min}$. The shortest interview at $6 \mathrm{~min}$ was the result of the participant being called away to attend to patient care. Participants completed a short demographic questionnaire prior to the start of the interview. Data saturation occurred once data findings were noticeably redundant despite new participants. 


\section{Data analysis}

Directed content analysis was used to analyse the transcribed interviews. ${ }^{18}$ Each transcript was individually reviewed and coded by the principal investigator (PI) and two research staff. Simultaneous and iterative coding occurred with the patient, caregiver and healthcare professional interviews. The initial coding schema, derived from the data, was developed once consensus was met between the coders. The codes were combined into categories that were further refined into themes by the PI and two research staff. As a final step to ensure methodological rigour, the PI reviewed all of the original transcripts with the emergent coding schema to ensure all codes and categories had been captured from the transcripts in the final coding schema. ${ }^{16}$

\section{RESULTS}

\section{Participant characteristics}

In total, 15 patients from an orthopaedic unit who met the eligibility criteria were identified, and 13 patients consented to participate. Two were screened and found to be ineligible with one patient subsequently transferred to a non-rehabilitation unit and the other presented with a cognitive deficit. Cognitive deficit was determined based on recent cognitive testing as reported in the patient's chart. Patient participants were mostly female $(n=9)$ with four males. The patients had an average age of 82.9 years with a range from 68 to 91 . Of the 13 patients who were enrolled in the study, nine caregivers were recruited and enrolled in the study. Caregiver participants reported an average age of 63.1 years, which ranged from 51 to 89 years. In total, 50 healthcare professionals participated in an interview with 29 nurses, six pharmacists, eight physical therapists, three social workers, two occupational therapists, one patient care manager and one physician. There were 39 female and 11 male study participants in this cohort. One healthcare professional approached for the study refused to participate because of concerns related to anonymity. Table 1 provides more details on the study participant characteristics.

\section{FINDINGS}

The following three themes were identified from the interviews: having no clue what the care plan is, being told and notified about the plan and experiencing challenges absorbing information.

\section{Having 'no clue' what the care plan is}

This theme reflects how several patients and their caregivers were unaware of the details of their transition plan from the acute care to rehabilitation hospital setting. Several participants described having 'no clue' about their care plan. Caregiver and patient experiences ranged from receiving 'tidbits of information' to 'no information' and 'leaving without a plan' when the patient was being transferred. As a result, some caregivers described not knowing what to expect at the rehabilitation hospital. For example, one caregiver described being 'plunked' in the hospital with a package of papers in an envelope that contained information on follow-up appointments that were not explained to them. Healthcare professionals acknowledged that they often did not know if the transition plan had been communicated to patients and

Table 1 Study participant characteristics

\begin{tabular}{|c|c|}
\hline Characteristic & Description \\
\hline \multicolumn{2}{|l|}{ Patients } \\
\hline Morbidities & $\begin{array}{l}\text { Patients had on average } 5.4 \text { morbidities (eg, Parkinson's disease, chronic obstructive pulmonary } \\
\text { disease, hypertension), which ranged from } 2 \text { to } 16\end{array}$ \\
\hline Living arrangements & All patients lived alone with the exception of one patient who lived with a spouse \\
\hline Medications & The patients were prescribed on average 8.5 medications with a range of $5-17$ medications \\
\hline \multicolumn{2}{|l|}{ Caregivers } \\
\hline Relationship to patient & Seven were adult children, one was a spouse and one was a sibling \\
\hline Duration of caregiver role & 6.25 on average with a range of $1-20$ years \\
\hline \multicolumn{2}{|l|}{ Healthcare professionals } \\
\hline Years of experience & $\begin{array}{l}\text { Less than } 1 \text { year } n=3 \\
2-5 \text { years } n=14 \\
6-10 \text { years } n=6 \\
11-15 \text { years } n=12 \text {, more than } 16 \text { years } n=15\end{array}$ \\
\hline Job status & $\begin{array}{l}\text { Full time }(n=39) \\
\text { Part time }(n=11)\end{array}$ \\
\hline Level of education & $\begin{array}{l}\text { Baccalaureate } n=24 \\
\text { Graduate } n=17 \\
\text { College } n=9\end{array}$ \\
\hline
\end{tabular}


their caregivers. This theme is illustrated in the following quotes:

I just assume that somewhere along the way I was going to get out. Then it was 1 day, you're going tomorrow and I thought, really? Already? And off we went. I don't recall anyone saying, when you get there, you can expect this to be different or that to be the same or ... it was just, okay, now is the time and off you go. (patient hospital site 2)

I was given a package with her follow-up appointments in it but it wasn't explained to me. So I have no clue How does she get there? Is anything happening over the weekend? Is it starting on Monday? What's going to happen? No information. We've just been plunked here with no information. (caregiver hospital site 1)

It was to my surprise yesterday, that I received a call from one of her visitors telling me that your mom is going to Hospital-X tomorrow and that she needs clothes. I think that I should have received a more formal call from somebody with the plan. There are questions, little tidbits of information I have received, that is, mom's current drugs that she's taken. So apparently that's being changed...I don't know the outcome of anything so I don't know the plan except that she's here now at Hospital-X. (caregiver hospital site 2)

They have a discharge list, a little envelope that the patients get. I'm not sure that they go through all of that with the patient. I think it probably varies. I don't know. I'm going to guess that some nurses are really good and go over it in detail, and I think others just say, here's your envelope, goodbye. (physician rehabilitation hospital)

Caregivers also described that they would have to wait to connect with healthcare professionals to engage in a conversation around the care transitions plan and goals for their family member who was a patient. In some cases, caregivers questioned whether there was a plan in place that had not been communicated to them or their family member. Healthcare professionals described withholding details of the plan until they themselves knew where the patient was going to and what would be happening next. For some participants when they did speak to healthcare professionals, they described receiving information around the immediate status of their family member but minimal to no information about the follow-up plan. One caregiver described that the healthcare professionals were 'dealing with the stage you are at' and one healthcare professional described 'being focused on doing the rehab part'. The following narrative examples elucidate this theme.

No one has spoken to me or to my mother about the goal. No one's talked about a deadline or time period, which they tell you in the booklet they will. So I keep waiting for someone to call me so we can sit down and talk about this. I still have no idea how long my mother will be here. It seems that they deal with the stage you're at. Hospital- $X$ was an acute care, just to deal with the pain management, to start physiotherapy and this is the next stage in the physiotherapy, more intense physiotherapy. In terms of follow-up or whether there will be followup, nothing. In terms of being put in communication as to where my mother is going, there's been no connection whatsoever. (caregiver hospital site 1)

I think families and patients have expectations that they want to go to a certain rehab facility, but that doesn't always happen. I don't know if that is always carried out clearly to the patient and their respective family members. I would say I don't tell them consistently ... I would tell them if they asked me and it doesn't come up naturally. (occupational therapist hospital site 1)

\section{Being told and notified about the plan}

This theme captures how some patients and their caregivers were informed about their plan for care transitions. Experiences of being informed of the care transitions plan mainly were one direction in nature in that healthcare professionals told and explained the plan to patients and/or their caregivers but did not engage in an interactive dialogue. For some participants, healthcare professionals explained what was going on and where the patients were going with the patients and their caregivers. This often included telling the patients and caregivers information about follow-up appointments, expectations about their recovery and therapy to where they were being transferred to and discharged home, and, in some cases, equipment that will be required once discharged home. Patients and caregivers did not report being engaged in an active discussion with healthcare professionals about their care transitions plan. For example, one caregiver and patient described being 'well informed all the time' but not being involved in a discussion with the healthcare professionals about their care transitions plan. Often patients and caregivers were comfortable with the passive nature of information exchange as they felt that the healthcare team knew what they were doing and would connect with patients and their caregivers at their discretion when required. This theme is further illustrated in the following quotes:

Hospital-X did a really, really good job of keeping all of us informed and relaying whatever information they had, they would give it. I certainly knew all that was going on. I was well aware of the process. (patient hospital site 1)

It was more just us expressing our recommendations, and the patient agreeing, and then we moved forward with the plan just by submitting it. So I think, probably the patient's role is also minimal once we've determined, yes, rehab is the plan. So other than consenting and choosing his preferences, I think 
their involvement in transitioning from here to rehab is also probably minimal. (physiotherapist hospital site 1)

Most of the time, the patient comes here, they're expecting to have their lives back, to do whatever they do before this fracture, these things happened. Now that you ask it, some of the patients don't know what the expectation is from after surgery and going to a different facility. Because they think that's like when you have hip surgery or something like that, they only stay for 24 hours and then go to another facility. But those patients that are first time, seriously they don't know. So that's why when they come here, we have to explain. (nurse rehabilitation hospital)

It was all in the folder that she got, and I was reading through it before we even left the hospital. I knew what to expect when we left the hospital. I knew everything ahead of time so I was up on all of it because the appointments and everything that were scheduled for her were all in the envelope. Everything was there, and it was all well put together. (caregiver hospital site 1, discharge interview)

\section{Experiencing challenges absorbing the information}

This third theme elucidates that some patients experienced challenges when attempting to absorb the information that was provided to them about their care transition plan. Challenges in absorbing the information were often associated with the patients being 'quite medicated' and 'drugged up' or experiencing symptoms (eg, pain and constipation) that compromised their ability to understand the information. The fast pace of recovering from a non-elective injury and 'getting bombarded' with a number of healthcare professionals was also reported by patients and their caregivers. In turn, this pace and number of interactions with healthcare professionals were perceived as contributing to patients not being able to remember and absorb information about what was happening and their care transitions plan. The following excerpts are examples of this theme:

I have to say that, while I was at Hospital-X, I was quite heavily medicated because of just having had the hip replacement done. I wouldn't have had a clue what was happening about half the time, not because people weren't telling me but just because I wouldn't have been able to keep up with them. (patient hospital site 2)... As mom says, things were coming like a train down a track. Everything was moving at high speed. There is important information that is coming to you all of the time and it's coming at you fast and furious. (caregiver hospital site 2)

I think when our nurse practitioner and case manager speaks to family or a patient about the next step in their plan. I'm sure they are being conveyed, but sometimes, when everything is happening so quickly, a patient might have forgotten what is going on or the family might be a little bit confused. (occupational therapist hospital site 1)

It's a little bit hard in the first day because they either travelled, they're tired, they're in pain, so they're not quite there and they get bombarded with everyone talking to them on the first day. (pharmacist rehabilitation hospital)

But Mom, I don't think was in any position to absorb the amount of information and the decision making. They can't just flit in and out of her bedroom with tons of information and expect her to retain it. (caregiver hospital site 2)

\section{DISCUSSION}

Our findings elucidate the nature of the exchange of information among patients and their caregivers with healthcare professionals during transitions from acute care hospitals to rehabilitation settings. The three emergent theme findings add to the growing body of literature regarding information exchange during care transitions from acute care hospital to rehabilitation settings.

The experience of not knowing the plan and confusion about next steps (having 'no clue') identified in our study was consistent with what has been reported in other studies. ${ }^{781219-21}$ Our finding of patients and their caregivers having tidbits of or no information is in line with other studies. For example, one study reported that as patients transferred from the hospital to a rehabilitation setting, they received very little information about what happened to them, and when they did, it was typically unstructured or insufficiently explained. ${ }^{8}$ Likewise, another study reported that patients and caregivers did not receive the required information about the reasons for their transfers to hospitals, medical diagnoses and planned treatments, resulting in lack of awareness of both their change in health status and transition plan. ${ }^{19}$ In our study, the lack of information resulted in confusion for patients and caregivers with little guidance on how to navigate post transfer from the hospital. In another study, more than one-third of patients could not clearly describe their diagnosis, and less than half could recall follow-up appointments that had been made for them. ${ }^{21}$

Our study finding regarding patients and caregivers waiting to connect with healthcare professionals to find out information on the care transition plan has been reported elsewhere. ${ }^{19}$ Similar to our study, others have reported not talking to anyone about their postdischarge care. ${ }^{12}$ In our study, when an information exchange did occur between patients and their caregivers with healthcare professionals, the exchange was often uni-directional (eg, patients and caregivers were told, informed, notified). Furthermore, the exchange focused on the immediate status of the patient compared with the overall plan for transitions from the hospital to the rehabilitation hospital to eventually being discharged home or to a long-term care facility. Interesting, other studies have described patients and caregivers as satisfied with the nature of a 
uni-directional information exchange ${ }^{11}$ and not viewing it necessary to obtain more information than what was provided. ${ }^{8}$

Our study finding around the challenges that patients experienced in absorbing information about their plan for transitions to the rehabilitation setting has also been highlighted in research focused on discharge home from hospital. ${ }^{9}$ Similar to the findings of Coleman $e t a l,{ }^{2}$ the volume of information and number of interactions with healthcare professions conveyed in a short period of time presented significant challenges to patients being able to absorb and retain the information around their care transition plan.

Collectively, our study findings elucidate the need for a multicomponent approach to ensure effective information exchanges are occurring with patients and their caregivers as they transition from acute care hospital to rehabilitation settings. Providing information to patients and their caregivers in a timely fashion and with consistent healthcare professionals is essential to ensure that they understand the information provided to them. Patients and caregivers should be kept thoroughly informed throughout the various care transition points. ${ }^{20}$ Healthcare professionals need to ascertain what information the patient and caregiver want to know and when and how and when to best to convey this relevant information to ensure adequate understanding of and carrying out of their care transitions plan. ${ }^{7}$ Furthermore, healthcare professionals need to determine whether patients and caregivers prefer to be passively or actively involved in their care transition planning. ${ }^{14}$

Recently, in Ontario, the implementation of a patient-oriented discharge summary (PODS) tool with a variety of patient populations being discharged from hospital to home has shown that the majority of patients who receive PODS have an improved understanding of their discharge instructions. ${ }^{22}{ }^{23}$ This tool could be adapted for interfacility care transitions, and study findings could assist in tailoring the tool to the local context and ensure that accurate, timely and relevant information is exchanged during interfacility transitions of care. For example, the interfacility care transition tool could have information on the care transition plan on where the patient is being transferred to, follow-up appointments, who to contact should worsening symptoms occur, medications, and any other information deemed relevant and meaningful for the patient and caregiver to have included.

This tool should be completed as part of a face-to-face conversation that healthcare professionals engage in with patients (and their caregivers when present) around the care transition plan. Furthermore, promising signs are being observed with teach-back methods whereby patients are provided information about the care transition plan including where the patient is going to by healthcare professionals and are then asked to share what information has been provided to them. ${ }^{121}$ This strategy allows for the healthcare professional to verify the patients' (and when present the caregiver(s)) understanding of the care transition plan and correct any misinterpretations. ${ }^{19}$ This type of information exchange is particularly important for patient populations (like the older non-elective orthopaedic patient included in this current study) who experience challenges absorbing information.

Finally, the Registered Nurses Association of Ontario's Care Transitions Best Practice Guideline provides a series of evidence and expert informed recommendations to ensure that effective information exchange among patients, their caregivers and healthcare professionals occurs during care transitions. ${ }^{24} 25$ Furthermore, a recent realist review and structured expert panel identified a series of interventions aimed at enhancing care transitions at the system, organisational, healthcare professions/teams and patient/caregiver levels. ${ }^{17}$ Together, these data sources can be used as blueprints by leaders in their efforts of creating and enacting policies and a cultivating a culture of caring aimed at improving the information exchange as a mechanism to enhance the quality of the relationship between patients and caregivers and healthcare professionals during care transitions.

Our study findings need to be viewed with the following potential limitations. First, our findings may not be transferrable to other geographical locations or other patient populations (eg, medically complex patients without orthopaedic injury). Second, there is a potential for social desirability bias when conducting interviews with participants. To mitigate this bias, the interview guide was carefully constructed to elicit patients', their caregivers' and professionals' perceptions and experiences associated with the care transitions from acute care hospital to a rehabilitation hospital.

\section{CONCLUSION}

Our study provides insight around the nature of information exchange with patients as they transition from acute care hospital to rehabilitation settings. Specifically, our study revealed that many patients and caregivers have little to no information about their plan and are waiting to get this information so they know what to expect. When patients and caregivers received information, it was a passive exchange where they were told or notified of what was going on and what was next representing a more paternalistic as opposed to collaborative exchange. For some patients, they had challenges absorbing the information provided due to being medicated, experiencing other symptoms or being approached by too many healthcare professionals. Future efforts are required to ensure that effective information exchanges are occurring with patients and their caregivers as they transition from acute care to rehabilitation to ensure that information is shared and discussed with patients and caregivers. Bidirectional exchange among professionals, patients and, where possible, caregivers will provide opportunities for all parties to provide input into the plan and confirm 
understanding and expectations. Furthermore, choosing an appropriate time to share the information, where possible, when the patient is most stable, will ensure a greater potential of information retention.

Contributors LJ conceived and designed the study. MS, ML, KK and SE planned and completed the analysis. LJ wrote the manuscript with revisions being made by MS, ML, KK and SE. All authors read and approved of the final manuscript.

Funding This project was funded by the Ontario Ministry of Health and Long Term Care.

Competing interests None declared.

Patient consent Consent was obtained using approved informed consent forms by the associated REBs.

Ethics approval St. Michael's Hospital Research Ethics Board and the Sinai Health System Research Ethics Board.

Provenance and peer review Not commissioned; externally peer reviewed.

Data sharing statement There is no additional data available.

Open Access This is an Open Access article distributed in accordance with the Creative Commons Attribution Non Commercial (CC BY-NC 4.0) license, which permits others to distribute, remix, adapt, build upon this work non-commercially, and license their derivative works on different terms, provided the original work is properly cited and the use is non-commercial. See: http://creativecommons.org/ licenses/by-nc/4.0/

(C) Article author(s) (or their employer(s) unless otherwise stated in the text of the article) 2017. All rights reserved. No commercial use is permitted unless otherwise expressly granted.

\section{REFERENCES}

1. Coleman EA, Chugh A, Williams MV, et al. Understanding and execution of discharge instructions. Am J Med Qual 2013;28:383-91.

2. Coleman EA. Falling through the cracks: challenges and opportunities for improving transitional care for persons with continuous complex care needs. J Am Geriatr Soc 2003;51:549-55.

3. Olsen RM, Østnor BH, Enmarker I, et al. Barriers to information exchange during older patients' transfer: nurses' experiences. J Clin Nurs 2013;22:2964-73.

4. King BJ, Gilmore-Bykovskyi AL, Roiland RA, et al. The consequences of poor communication during transitions from hospital to skilled nursing facility: a qualitative study. J Am Geriatr Soc 2013:61:1095-102.

5. Burbach BE, Cohen MZ, Zimmerman LM, et al. Post-hospitalization transition to home: patient perspectives of a personalized approach. J Nurs Educ Pract 2016;6:32-40.

6. Dossa A, Bokhour B, Hoenig H. Care transitions from the hospital to home for patients with mobility impairments: patient and family caregiver experiences. Rehabil Nurs 2012;37:277-85.
7. Jeffs L, Kitto S, Merkley J, et al. Safety threats and opportunities to improve interfacility care transitions: insights from patients and family members. Patient Prefer Adherence 2012;6:711-8.

8. McKain S, Henderson A, Kuys S, et al. Exploration of patients' needs for information on arrival at a geriatric and rehabilitation unit. J Clin Nurs 2005;14:704-10.

9. Ford BK, Ingersoll-Dayton B, Burgio K. Care transition experiences of older veterans and their caregivers. Health Soc Work 2016;41:129-38.

10. Strahan $\mathrm{EH}$, Brown RJ. A qualitative study of the experiences of patients following transfer from intensive care. Intensive Crit Care Nurs 2005;21:160-71.

11. Flink M, Öhlén $\mathrm{G}$, Hansagi $\mathrm{H}$, et al. Beliefs and experiences can influence patient participation in handover between primary and secondary care - a qualitative study of patient perspectives. BMJ Qual Saf 2012;21:i76-i83.

12. Flacker J, Park W, Sims A. Hospital discharge information and older patients: do they get what they need? J Hosp Med 2007;2:291-6.

13. Dyrstad DN, Laugaland KA, Storm M. An observational study of older patients' participation in hospital admission and dischargeexploring patient and next of kin perspectives. $J$ Clin Nurs 2015;24:1693-706.

14. Swinkels A, Mitchell T. Delayed transfer from hospital to community settings: the older person's perspective. Health Soc Care Community 2009; $17: 45-53$

15. Gandara E, Moniz T, Ungar J, et al. Communication and information deficits in patients discharged to rehabilitation facilities: an evaluation of five acute care hospitals. J Hosp Med 2009;4:E28-E33.

16. Patton MQ. Qualitative research and evaluation methods. California: Thousand Oaks, 2002.

17. Jeffs L, Kuluski K, Law M, et al. Identifying effective nurse-led care transition interventions for older adults with complex needs using a structured expert panel. Worldviews Evid Based Nurs 2017;14:136-44.

18. Hsieh HF, Shannon SE. Three approaches to qualitative content analysis. Qual Health Res 2005;15:1277-88.

19. Toles MP, Abbott KM, Hirschman KB, et al. Transitions in care among older adults receiving long-term services and supports. J Gerontol Nurs 2012;38:40-7.

20. Rustad EC, Furnes B, Cronfalk BS, et al. Older patients' experiences during care transition. Patient Prefer Adherence 2016;10:769-79.

21. Horwitz LI, Moriarty JP, Chen C, et al. Quality of discharge practices and patient understanding at an academic medical center. JAMA Intern Med 2013;173:1715-22.

22. Hahn-Goldberg S, Okrainec K, Huynh T, et al. Implementing patient oriented discharge summaries (PODS): a multi-site pilot across early adopter hospitals. Accepted to Healthcare Quarterly. In Press. 2015.

23. Hahn-Goldberg S, Okrainec K, Huynh T, et al. Co-creating patientoriented discharge instructions with patients, caregivers, and healthcare providers. J Hosp Med 2015;10:804-7.

24. Dusek B, Pearce N, Harripaul A, et al. Care transitions: a systematic review of best practices. J Nurs Care Qual 2015;30:233-9.

25. Registered Nurses Association of Ontario. Care transitions Best Practice Guideline. http://rnao.ca/sites/rnao-ca/files/Care Transitions_BPG.pdf. (accessed Aug 2016). 